\title{
La administración del transporte urbano: alternativa de uso sustentada en la educación sostenible
}

\author{
Management of Urban Transportation: Alternative of Use Based on \\ Sustainable Education
}

A gestão do transporte urbano: alternativa de uso apoiada na educação sustentável

Laura Vázquez Nájera

Instituto Politécnico Nacional, México

lau_vn@yahoo.com https://orcid.org/0000-0003-3136-976X

Ma. de los Ángeles Martínez Ortega Instituto Politécnico Nacional, México mmartinezo@ipn.mx https://orcid.org/0000-0002-8483-9844

Martha Jiménez García Instituto Politécnico Nacional, México majimenez@ipn.mx https://orcid.org/0000-0002-8556-2955 




Revista Iberoamericana para la Investigación y el Desarrollo Educativo ISSN $2007-7467$

\section{Resumen}

El objetivo de este trabajo fue determinar los motivos de la movilidad o desplazamiento de habitantes de la Ciudad de México y especificar el propósito del viaje y el tipo de transporte utilizado, así como observar de manera directa el involucramiento de la mujer en todo ello. Asimismo, con la información anterior, diseñar una propuesta de administración del transporte público y la movilidad de los usuarios para optimizar sus tiempos y movimientos. Para lo anterior, se tomaron datos de la Encuesta Origen Destino en Hogares de la Zona Metropolitana del Valle de México (EOD) 2017 del Instituto Nacional de Estadística y Geografía [Inegi] (2017). La muestra fue de 160095 personas. Se realizó la prueba del coeficiente de Pearson para determinar el grado de correlación entre las variables relacionadas con la movilidad. Los resultados reflejaron lo siguiente: el género (0.8865) se relaciona con el propósito de ir al hogar (0.9200), ir al trabajo (0.8967), al lugar de estudio (0.9971), para ir de compras (0.7898), al lugar de convivencia (0.9528), para llevar a alguien a un destino (0.7282), para realizar trámites $(0.9808)$, para acudir a un recinto de salud (0.8944) y para acudir a un lugar religioso (0.8115). Se concluye que la correlación más alta es en el desplazamiento para ir a estudiar; enseguida, para acudir a lugares de convivencia, y un tercer motivo, dirigirse al hogar. Finalmente, se propone considerar la formación humana e incidir de manera directa desde la educación para formar agentes de transformación y lograr una administración eficiente del transporte público.

Palabras clave: gestión, motivos de desplazamientos, movilidad urbana, servicios de movilidad, sustentabilidad.

\section{Abstract}

The objective of this work was to determine the reasons for the mobility or displacement of the inhabitants of Mexico City and to specify the purpose of the trip and the type of transport used, as well as to directly observe the involvement of women in all this. Likewise, with the above information, design a proposal for the administration of public transport and the mobility of users to optimize their times and movements. For the above, data were taken from the Encuesta Origen Destino en Hogares de la Zona Metropolitana del Valle de México (EOD) 2017 of the Instituto Nacional de Estadística y Geografía [Inegi] (2017). The sample was 160095 people. The Pearson coefficient test was performed to determine the degree of 


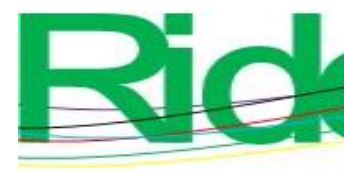

Fecha Recepción: Noviembre 2020
Revista Iberoamericana para la Investigación y el Desarrollo Educativo ISSN $2007-7467$

Fecha Aceptación: Mayo 2021

\section{Introducción}

Para la Organización de las Naciones Unidas para la Educación, la Ciencia y la Cultura (Unesco) (Leicht, Heiss y Byun, 2018), la educación es una prioridad universal. Se trata de un derecho humano básico que facilita la construcción de un estadio de paz, impulsa el desarrollo sostenible y responde a los desafíos actuales con un enfoque en la igualdad de género.

En el Plan Nacional de Desarrollo 2018-2024, en su objetivo 3.6, propone desarrollar, de manera transparente, una red de comunicación y transporte accesible, segura, eficiente, sostenible, incluyente y moderna, con visión de desarrollo regional y de redes de logística que conecte a todas las personas, facilite el traslado de bienes y servicios y que contribuya a salvaguardar la seguridad nacional, de acuerdo con el Centro de Estudios de las Finanzas Públicas [CEFP] (2019). De manera específica, como una estrategia puntual, señala contar con una red carretera segura y eficiente que conecte centros de población conservando su valor patrimonial (CEFP, 2019).

Para el diseño de una propuesta asertiva, es necesario tomar como referencia la normatividad vigente. En este caso, la Ley de Movilidad de la Ciudad de México, en su artículo LV, define movilidad de la siguiente forma:

Conjunto de desplazamientos de personas y bienes que se realizan a través de diversos modos de transporte, que se llevan a cabo para que la sociedad pueda satisfacer sus necesidades y acceder a las oportunidades de trabajo, educación, salud, recreación y demás que ofrece la Ciudad (H. Congreso de la Ciudad de México I Legislatura, 23 de abril de 2020, p. 6).

Kaufmann y Widmer (2006) consideran que el concepto de motilidad es una excelente base para analizar las motivaciones de las personas, el proceso de toma de decisiones y las constricciones que dominan el uso del espacio. La motilidad es definida como la forma en la que un individuo o un grupo de personas toma posesión del reino de las posibilidades de movilidad y hace uso de ellas. Así, la movilidad urbana también se vincula con la capacidad o el potencial para moverse dentro de una localidad. Si bien los avances sociales y tecnológicos continuamente están modificando dichas posibilidades de movilidad de las personas, es imprescindible trasladarse de un lugar a otro para acceder a diferentes servicios, 


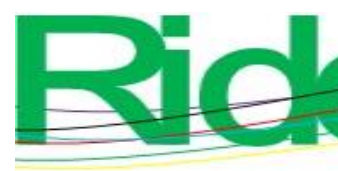

Revista Iberoamericana para la Investigación y el Desarrollo Educativo ISSN $2007-7467$

algunos básicos, como lo son la sanidad y la educación, y otros no tan imprescindibles pero también necesarios, como el ocio (Montouto y Yustos, 2010).

La movilidad y el desplazamiento del ser humano es una condición natural para ver satisfechas varias necesidades. La movilidad es una acción o práctica social de desplazamiento en el territorio. Se trata de un concepto que no se reduce al transporte y contiene un modo de interpretar el acto de desplazamiento (Gutiérrez, 2012).

Otro concepto importante para los fines de este trabajo es la movilidad sostenible. El conjunto de acciones orientadas al logro del uso racional de los medios de transporte por parte de los ciudadanos, así podría definirse. Para ello, es necesario conseguir espacios más habitables y saludables y poder avanzar hacia un modelo social en donde la comunidad, las personas y su entorno dominen sobre la movilidad cotidiana y su impacto; espacios urbanos e interurbanos de calidad, con condiciones de accesibilidad y optimización del tiempo, que impacten de manera positiva en el entorno social, laboral o personal (Montouto y Yustos, 2010).

La pretensión principal de esta investigación fue identificar los propósitos principales de los viajes que realizan los habitantes de la zona metropolitana del Valle de México (ZMVM) y el tipo de transporte utilizado. En otras palabras, analizar la movilidad o desplazamiento del individuo y especificar el propósito del viaje, el tipo de transporte utilizado y observar de manera directa el involucramiento de la mujer en todo ello, con miras a diseñar una propuesta de administración y planeación eficaz del transporte público capaz de optimizar tiempos y movimientos. En suma, proponer una alternativa de solución a la saturación del transporte, principalmente del transporte público.

Se parte de la siguiente pregunta de investigación: ¿la identificación de los propósitos de los viajes que realizan los individuos en la movilidad urbana cotidiana en la ZMVM, el análisis de esos motivos y su correlación con el género facilitarán el proceso de la administración del transporte?

Se planteó una hipótesis nula que señala que no existe una correlación de los motivos de desplazamiento con la movilidad urbana cotidiana de los ciudadanos de la ZMVM.

A continuación, se presenta el método de análisis. Inmediatamente después, se muestran y se relacionan los resultados con los fundamentos teóricos. Más tarde, se señala una propuesta de planeación. Y finalmente, se llega a las conclusiones. 


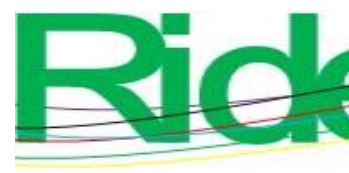

Revista Iberoamericana para la Investigación y el Desarrollo Educativo ISSN $2007-7467$

\section{La movilidad urbana y sus principales motivos}

\section{La movilidad urbana}

De acuerdo con la Real Academia Española [RAE] (2020), la movilidad es la cualidad, capacidad o posibilidad de moverse o desplazarse. Teniendo en cuenta lo anterior, la movilidad urbana podría considerarse como la capacidad de desplazarse o moverse dentro de un espacio urbano. Respecto a la movilidad urbana inteligente, es aquella conectividad entre pueblos y ciudades asequible, eficaz, atractiva y sostenible que vincula lo inteligente y lo sostenible (Lyons, 2018).

Una de las consecuencias del crecimiento urbano ha sido un mayor desplazamiento humano para poder realizar actividades consideradas necesarias para subsistir. Ahora bien, no hay que perder de vista que las características individuales de las personas están estrechamente relacionadas con su capacidad de movimiento. Y que la infraestructura y la suficiencia del transporte no bastan para hablar de individuos más móviles; se tienen que considerar otros factores (Aparicio, 2018).

\section{La movilidad por género}

Es de suma importancia conocer los motivos que generan los desplazamientos cotidianos de las mujeres debido a que en ocasiones las estrategias de movilidad manifiestan desigualdades de género (Riquelme, 2016).

Los desplazamientos de las mujeres por la ciudad no son uniformes. La heterogeneidad de movilidades está en función del entorno próximo, la experiencia vital y la vinculación con los servicios ofertados (Riquelme, 2016). Es necesario resaltar que las desigualdades de movilidad de género en la ZMVM son más acentuadas en contraste con las detectadas en Europa (Pérez y Capron, 2018).

\section{La movilidad para dirigirse al hogar}

El lugar de localización de la residencia es muy importante para decidir la planeación de la movilidad. Allí donde tiene lugar la socialización territorial existe una relación con la movilidad social porque es donde viven las familias y está asociado con estilos de vida, lo cual se constituye como un elemento diferenciador entre las personas para decidir cómo moverse, cuánto presupuesto asignar y el tiempo idóneo; es decir, los recursos necesarios y variados para realizar el desplazamiento requerido. 


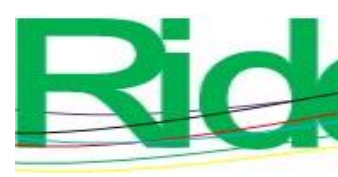

Revista Iberoamericana para la
Investigación y el Desarrollo Educativo
ISSN $2007-7467$

La movilidad es una condición clave de acceso al mercado laboral, a una vivienda, a la educación, a la cultura y el ocio, a la familia (Boniolo y Estévez, 2017). El derecho a trabajar, a tener una vivienda, a recibir capacitación implica el derecho a la movilidad. En cierto sentido, este derecho a la movilidad es una precondición de los otros derechos.

\section{La movilidad para dirigirse al trabajo}

La movilidad cotidiana de la población es generada y motivada por diversos propósitos: trabajar, estudiar, consumir, convivir, por mencionar algunos (Galindo, Pérez y Suárez, 2020). Un efecto directo que tuvo el incremento del número de empleos en el centro de México, específicamente en la ZMVM, fue el redireccionamiento de la movilidad cotidiana (flujos laborales) (Galindo et al., 2020).

\section{La movilidad para dirigirse al lugar de estudio}

Un estudio realizado en España manifiesta y resalta la importancia de educar para la sostenibilidad. Así, considerando el contexto social y administrativo, la movilidad ha adquirido un creciente protagonismo que involucra directamente a los miembros de la sociedad como agentes de cambio. Desplazarse hacia el área educativa ocupa un lugar muy considerable (Montouto y Yustos, 2010).

\section{La movilidad hacia lugares para realizar compras}

En un estudio realizado en cinco provincias de España se encontró que el desplazamiento al trabajo influye directamente en el resto de los motivos de su movilidad; acudir a espacios para realizar compras se ubicó en el lugar tercero (Montouto y Yustos, 2010).

\section{La movilidad para llevar al prójimo}

Las mujeres como generadoras de vida se dedican más que los hombres a las actividades de cuidado de los niños y también, en América Latina, de los ancianos (Pérez y Capron, 2018). Esto implica que, por lo general, sean ellas las que realicen las actividades de acompañamiento de los hijos a la escuela y a sus actividades periódicas (Pérez y Caprón, 2018). 


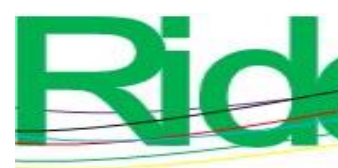

Revista Iberoamericana para la Investigación y el Desarrollo Educativo ISSN $2007-7467$

encuestas de viaje con la obtenida de tarjetas inteligentes utilizadas en sistemas de transporte. Con ayuda de mapas de uso de suelo y sistemas de información geográfica (SIG), identifican los patrones de las rutas de viajes pendulares, las formas de movilidad "obligatoria" que ocurren entre el domicilio y el lugar de estudio o trabajo (Gouëset, Demoraes, Le Roux, Figueroa y Zioni, 2015). Trabajos similares se han realizado en Seúl, Corea (Kim, Kim y Sohn, 2018) y en Shenzhen, China (Tang, Wang, Zong y Hu, 2020).

En esta investigación se propone que un sistema de planificación de recursos empresariales (ERP, por sus siglas en inglés) sea incluido como un sistema gestor que facilite la gestión de control de rutas del transporte. Un ejemplo vanguardista es el que actualmente utiliza el País Vasco. Gracias a un ERP para transporte, se ha reducido el riesgo en las operaciones de empresas de transporte urbano regular de pasajeros (TURP) (Grupo IGN, 2020).

\section{Administración eficaz del transporte urbano}

En la Ciudad de México existe un sistema de transporte público propuesto desde 2015, que se realizó para el control del servicio del transporte público concesionado. Para este programa se realizó un diagnóstico, un modelo de gestión, un análisis de costos y hasta se consideraron diversos escenarios de implantación. En ese momento, la situación del transporte público registra las siguientes cifras: $56 \%$ microbuses, $8.84 \%$ metrobús, $1.49 \%$ trolebús, $4.92 \%$ taxis, $24.35 \%$ Sistema de Transporte Colectivo-Metro (STCM) y el mexibús $4.21 \%$. La propuesta de implantación se dio por delegación, hoy alcaldías, a través de la administración puntual de los centros de transferencia modal (Cetram), por liderazgo, especificando módulos de ruta 100 y por líneas de metro, de acuerdo con CTS Embarq México (2015)

Sin duda, una de las principales tareas del gobierno de la CDMX es la promoción del uso del transporte público en términos de eficiencia y eficacia, y que no impacte de manera negativa en el medio ambiente. En un estudio realizado en 2018 para identificar las áreas de oportunidad que se tienen y para mejorar el servicio e incentivar su uso, los usuario manifestaron que los transportes analizados (metro, microbús y metrobús) no cubren sus expectativas de calidad, personalización y confiabilidad (Paredes y Palmer, 2018).

Resulta paradójico que existe una normatividad vigente, estudios y propuestas vanguardistas, pero sigue siendo un sistema insuficiente, ineficaz y desde luego ineficiente. 


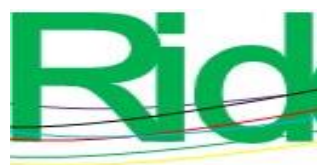

Revista Iberoamericana para la
Investigación y el Desarrollo Educativo
ISSN $2007-7467$

\section{Métodos y materiales}

Primeramente, se procedió con un enfoque cuantitativo y proceso deductivo. La investigación fue del tipo exploratoria, descriptiva y correlacional (Hernández, Fernández y Baptista, 2014). Para conocer la movilidad espacial cotidiana, la dirección de los flujos y el modo de transporte dividido por género de la ZMVM, se tomó como fuente directa la Encuesta Origen Destino en Hogares de la Zona Metropolitana del Valle de México (EOD) 2017 del Instituto Nacional de Estadística y Geografía [Inegi] (2017). Tanto la población como la muestra fueron definidas por el Inegi Unam. Para este estudio solo se tomaron datos de la población que realizaron movilidad en un primer tramo, considerando a 160095 personas. Como variable dependiente se consideró la movilidad y como variables independientes las siguientes: género, hogar, trabajo, lugar de estudio, sitio de convivencia, llevar a alguien, otro medio, auto, no sabe, otro motivo, ir a lugar religioso, ir a lugar de salud y para realizar trámites (figura 1). En la tabla 1 se describen las variables utilizadas en el modelo.

Figura 1. Variables de estudio del modelo

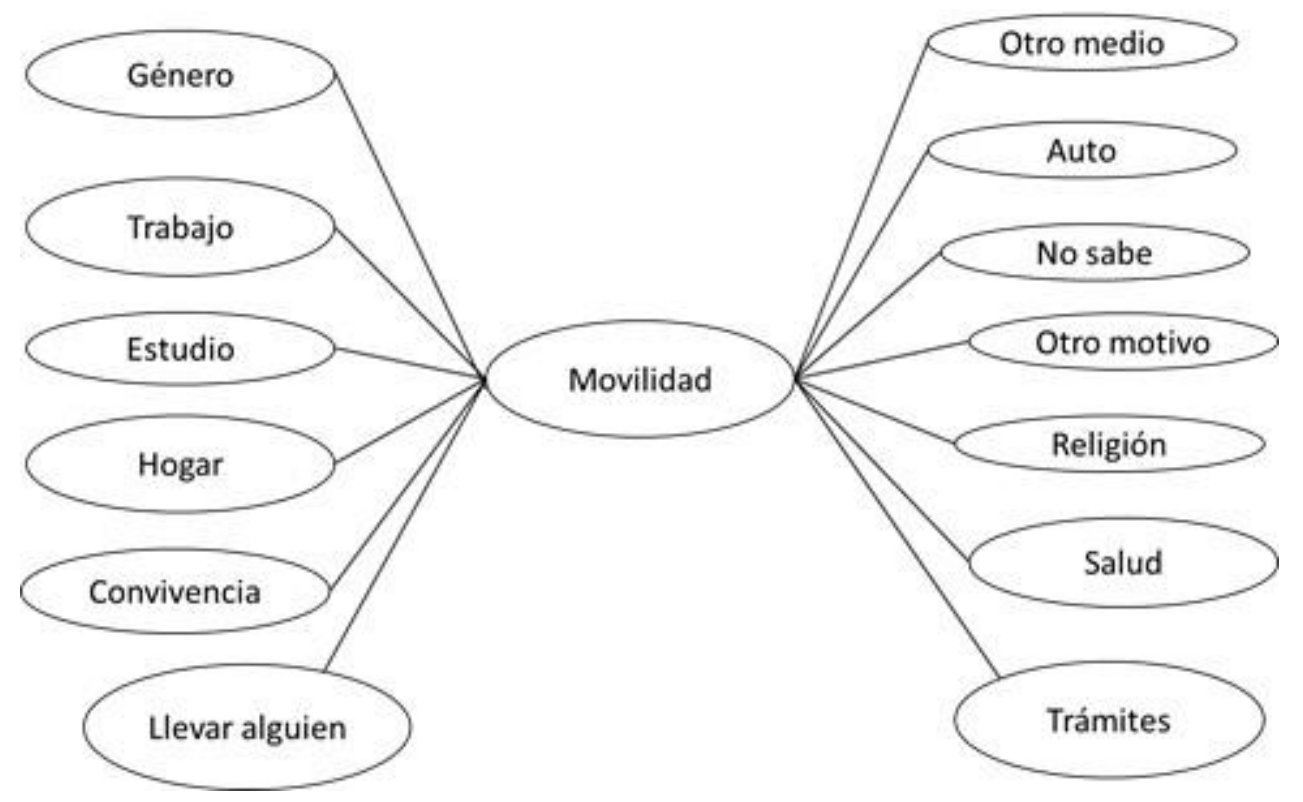

Fuente: Elaboración propia 


\section{Modelo}

Tomando como referencia las variables consideradas y vinculadas a la movilidad como propósito del viaje hecho por los usuarios, se realizó el análisis correlacional de Pearson, utilizando el software gretl versión 2016c.

El modelo se estableció considerando los coeficientes de correlación múltiple y usando las observaciones del uno al tres con un valor crítico de $5 \%$ (a dos colas), esto es, igual a 0.9969 para $n=3$. Si $r=0$ no existe correlación, si $r=1$ existe una correlación positiva. De igual manera, se siguió la siguiente fórmula:

$$
\begin{array}{cc}
\Sigma X Y= & \Sigma X \Sigma Y \\
r X Y=\sqrt{\left(\Sigma x^{2}-\left(\frac{\Sigma X}{n}\right)^{2}\right)} \sqrt{\left(\Sigma y^{2}-\left(\frac{\Sigma y}{n}\right)^{2}\right)}
\end{array}
$$




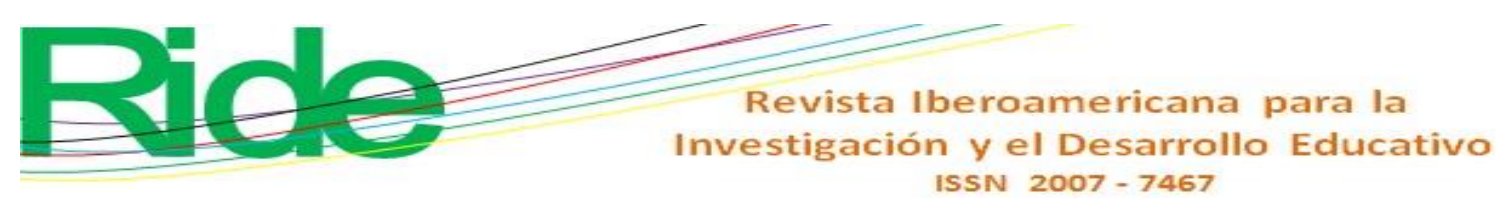

Tabla 1. Variables de estudio de movilidad

\begin{tabular}{|c|c|c|}
\hline Variable & Descripción & Tipo de variable \\
\hline Movilidad & $\begin{array}{l}\text { Propósito del viaje-considerado el motivo de } \\
\text { la movilidad o desplazamiento hacia el lugar } \\
\text { de destino. }\end{array}$ & $\begin{array}{l}\text { Dependiente: es el motivo para conocer } \\
\text { el propósito del desplazamiento del } \\
\text { individuo. }\end{array}$ \\
\hline Género & $\begin{array}{l}\text { En este caso se refiere a los usuarios hombres } \\
\text { y mujeres que realizaron viajes por diferentes } \\
\text { propósitos (variable cualitativa-dicotómica). }\end{array}$ & $\begin{array}{l}\text { Independiente: se relaciona con la } \\
\text { movilidad para determinarla por tipo de } \\
\text { persona que se desplaza (hombre-mujer). }\end{array}$ \\
\hline Hogar & $\begin{array}{l}\text { Número de usuarios que su destino fue llegar } \\
\text { a su domicilio (variable cuantitativa discreta). }\end{array}$ & \multirow{13}{*}{$\begin{array}{l}\text { Independientes: determinantes para } \\
\text { proponer la administración de los } \\
\text { movimientos o recorridos necesarios del } \\
\text { usuario y optimizar tiempos y } \\
\text { movimientos. }\end{array}$} \\
\hline Trabajo & $\begin{array}{l}\text { Número de usuarios que su destino fue llegar } \\
\text { a su lugar de trabajo (variable cuantitativa } \\
\text { discreta). }\end{array}$ & \\
\hline Estudio & $\begin{array}{l}\text { Número de usuarios que su destino fue llegar } \\
\text { a su lugar de estudio (variable cuantitativa } \\
\text { discreta). }\end{array}$ & \\
\hline Compras & $\begin{array}{l}\text { Número de usuarios que su destino fue llegar } \\
\text { a un lugar para realizar compras (variable } \\
\text { cuantitativa discreta). }\end{array}$ & \\
\hline Convivencia & $\begin{array}{l}\text { Número de usuarios que su destino fue llegar } \\
\text { a un lugar de recreación (variable cuantitativa } \\
\text { discreta). }\end{array}$ & \\
\hline $\begin{array}{l}\text { Llevar a } \\
\text { alguien }\end{array}$ & $\begin{array}{l}\text { Número de usuarios cuyo motivo de viaje fue } \\
\text { para llevar a alguien a un lugar determinado } \\
\text { (variable cuantitativa discreta). }\end{array}$ & \\
\hline Trámites & $\begin{array}{l}\text { Número de usuarios cuyo motivo de viaje fue } \\
\text { para realizar un trámite (variable cuantitativa } \\
\text { discreta). }\end{array}$ & \\
\hline Salud & $\begin{array}{l}\text { Número de usuarios cuyo motivo de viaje fue } \\
\text { para buscar atención médica (variable } \\
\text { cuantitativa discreta). }\end{array}$ & \\
\hline Religión & $\begin{array}{l}\text { Número de usuarios cuyo motivo de viaje fue } \\
\text { acudir a un lugar religioso (variable } \\
\text { cuantitativa discreta). }\end{array}$ & \\
\hline Otro & $\begin{array}{l}\text { Número de usuarios cuyo motivo de viaje fue } \\
\text { otro diferente a los señalados con antelación } \\
\text { (variable cuantitativa discreta). }\end{array}$ & \\
\hline No sabe & $\begin{array}{l}\text { Número de usuarios cuyo motivo de viaje no } \\
\text { fue especificado. }\end{array}$ & \\
\hline Auto & $\begin{array}{l}\text { Número de usuarios que utilizaron su } \\
\text { vehículo para transportarse (variable } \\
\text { cualitativa discreta). }\end{array}$ & \\
\hline Otro medio & $\begin{array}{l}\text { Número de usuarios que utilizaron medios } \\
\text { diferentes al auto para transportarse (variable } \\
\text { cualitativa discreta). }\end{array}$ & \\
\hline
\end{tabular}

Fuente: Elaboración propia con base en Inegi (2017) 

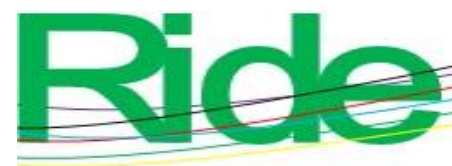

Revista Iberoamericana para la Investigación y el Desarrollo Educativo ISSN $2007-7467$

\section{Instrumento}

Se aplicó un cuestionario integrado por seis secciones: 1) Residentes y hogares, 2) Vehículos disponibles, 3) Características sociodemográficas (en este caso solo se consideró el género), 4) Identificación de las personas que viajaron, 5) Viajes realizados entre semana (en este caso solo se consideró la movilidad en el primer tramo de su recorrido una vez iniciado su viaje) y 6) Viajes realizados en sábado (no considerado).

El método de captación fue a través de dos entrevistas:

1) Informante de 15 años o más, de quien se obtuvo información de vivienda y sociodemográfica. Se le entregaron dos tarjetas de viajes a cada integrante de seis años o más.

2) Identificación de viajeros; se entrevistó individualmente a cada persona de seis años o más; con apoyo de las tarjetas de viaje, se respondió a las preguntas de las secciones restantes del cuestionario.

\section{Variable dependiente}

- $\quad$ Movilidad de las personas.

\section{Variables independientes}

- $\quad$ Tipo de vehículos utilizados.

- Género de los actores.

- $\quad$ Propósito del viaje.

\section{Resultados}

Respecto al análisis correlacional de Pearson, utilizando el software gretl versión 2016 c, el resultado que se obtuvo se observa en la tabla 2. 


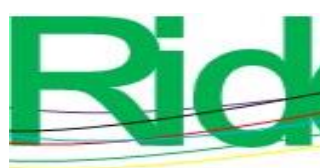

Revista Iberoamericana para la Investigación y el Desarrollo Educativo ISSN 2007 - 7467

Tabla 2. Resultados obtenidos a partir del análisis correlacional de Pearson

\begin{tabular}{|c|c|c|c|c|c|}
\hline Género & Hogar & Trabajo & Estudio & Compras & \\
\hline \multirow[t]{5}{*}{1.0000} & 0.6342 & 0.5901 & 0.8486 & 0.9840 & Género \\
\hline & 1.0000 & 0.9984 & 0.9472 & 0.4862 & Hogar \\
\hline & & 1.0000 & 0.9279 & 0.4367 & Trabajo \\
\hline & & & 1.0000 & 0.7406 & Estudio \\
\hline & & & & 1.0000 & Compras \\
\hline Convivencia & $\begin{array}{l}\text { Llevar } \\
\text { alguien }\end{array}$ & Trámites & Salud & Religión & \\
\hline 0.9851 & 0.9627 & 0.9598 & 0.9998 & 0.9898 & Género \\
\hline 0.7576 & 0.4013 & 0.8258 & 0.6475 & 0.5175 & Hogar \\
\hline 0.7201 & 0.3496 & 0.7931 & 0.6040 & 0.4690 & Trabajo \\
\hline 0.9269 & 0.6738 & 0.9630 & 0.8576 & 0.7645 & Estudio \\
\hline 0.9387 & 0.9955 & 0.8943 & 0.9807 & 0.9993 & Compras \\
\hline \multirow[t]{5}{*}{1.0000} & 0.9019 & 0.9937 & 0.9880 & 0.9506 & Convivencia \\
\hline & 1.0000 & 0.8480 & 0.9579 & 0.9914 & Llevar alguien \\
\hline & & 1.0000 & 0.9645 & 0.9099 & Trámites \\
\hline & & & 1.0000 & 0.9872 & Salud \\
\hline & & & & 1.0000 & Religión \\
\hline Otro & No sabe & Total lugar & Auto & Otro medio & \\
\hline 0.8651 & 0.7440 & 0.8865 & 0.6961 & 0.9186 & Género \\
\hline 0.9365 & 0.9885 & 0.9200 & 0.9966 & 0.8881 & Hogar \\
\hline 0.9155 & 0.9785 & 0.8967 & 0.9904 & 0.8611 & Trabajo \\
\hline 0.9995 & 0.9849 & 0.9971 & 0.9705 & 0.9886 & Estudio \\
\hline 0.7618 & 0.6130 & 0.7898 & 0.5570 & 0.8334 & Compras \\
\hline 0.9384 & 0.8478 & 0.9528 & 0.8091 & 0.9729 & Convivencia \\
\hline 0.6971 & 0.5355 & 0.7282 & 0.4759 & 0.7774 & Llevar alguien \\
\hline 0.9711 & 0.9017 & 0.9808 & 0.8697 & 0.9926 & Trámites \\
\hline 0.8737 & 0.7555 & 0.8944 & 0.7085 & 0.9253 & Salud \\
\hline 0.7848 & 0.6412 & 0.8115 & 0.5867 & 0.8529 & Religión \\
\hline 1.0000 & 0.9788 & 0.9990 & 0.9623 & 0.9929 & Otro \\
\hline
\end{tabular}


Revista Iberoamericana para la Investigación y el Desarrollo Educativo ISSN 2007 - 7467

\begin{tabular}{|c|c|c|c|c|c|}
\hline & 1.0000 & 0.9687 & 0.9976 & 0.9475 & No sabe \\
\hline & & 1.0000 & 0.9493 & 0.9972 & Total lugar \\
\hline & & & 1.0000 & 0.9232 & Auto \\
\hline & & & & 1.0000 & Otro medio \\
\hline & & & & Total medio & \\
\hline & & & & 0.8865 & Género \\
\hline & & & & 0.9200 & Hogar \\
\hline & & & & 0.8967 & Trabajo \\
\hline & & & & 0.9971 & Estudio \\
\hline & & & & 0.7898 & Compras \\
\hline & & & & 0.9528 & Convivencia \\
\hline & & & & 0.7282 & Llevar alguien \\
\hline & & & & 0.9808 & Trámites \\
\hline & & & & 0.8944 & Salud \\
\hline & & & & 0.8115 & Religión \\
\hline & & & & 0.9990 & Otro \\
\hline & & & & 0.9687 & No sabe \\
\hline & & & & 1.0000 & Total lugar \\
\hline & & & & 0.9493 & Auto \\
\hline & & & & 0.9972 & Otro medio \\
\hline & & & & 1.0000 & Total medio \\
\hline \multicolumn{6}{|c|}{ Coeficientes de correlación usando las observaciones 1-3 } \\
\hline Valor crítico a 5 & dos & $69 \mathrm{p}$ & & & \\
\hline
\end{tabular}

Fuente: Elaboración propia

Algo muy importante que se tiene que tener en cuenta es el tamaño de la muestra cuando se quiere apreciar la determinación del coeficiente en términos unitarios y porcentuales, ya que este último valor es muy importante, debido a que la interpretación de la correlación entre dos variables se realiza por medio del coeficiente de determinación como la variancia común de ambas. Mientras más grande es la muestra bastará que las variables tengan un mínimo de porcentaje en común para que exista una relación estadísticamente hablando significativa, sin embargo, pudiera ser que desde una óptica práctica este porcentaje sea poco relevante, ya que implicaría tener un alto porcentaje de variabilidad no compartida. 

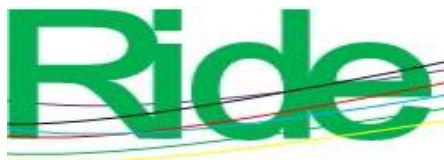

Revista Iberoamericana para la Investigación y el Desarrollo Educativo ISSN $2007-7467$

En la figura 2 se presentan los resultados de la movilidad por propósito del viaje y se percibe que el trabajo es el que ocupa el primer lugar, le siguen el estudio y después las compras.

Figura 2. Movilidad por propósito de viaje por género

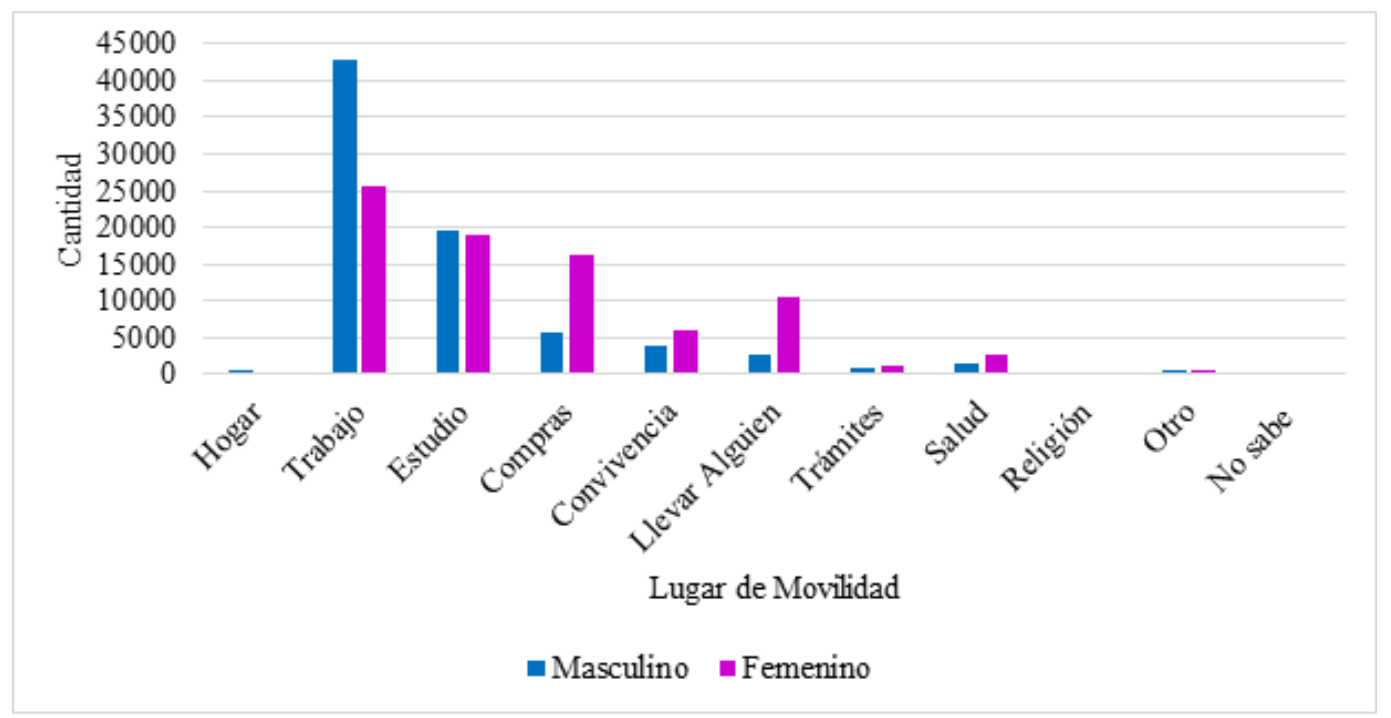

Fuente: Elaboración propia con base en Inegi (2017)

En la figura 3 se presenta la movilidad por tipo de transporte, y se muestra que la mayoría de la población la realiza por otro medio que no es el automóvil.

Figura 3. Movilidad por tipo de transporte

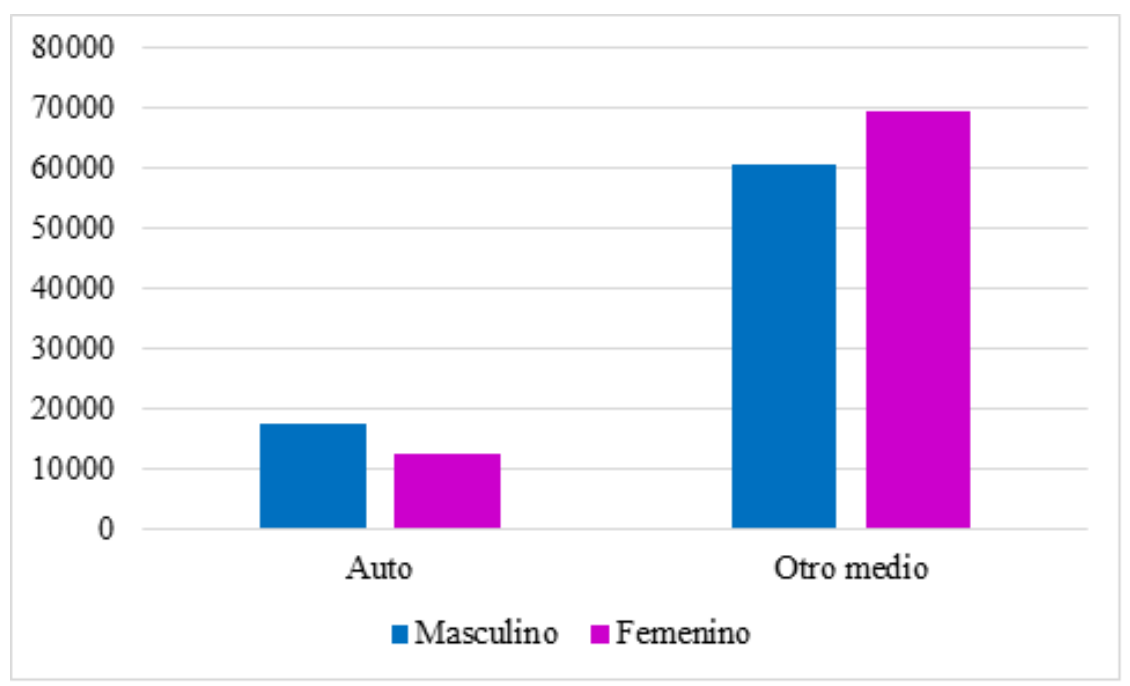

Fuente: Elaboración propia con base en Inegi (2017) 


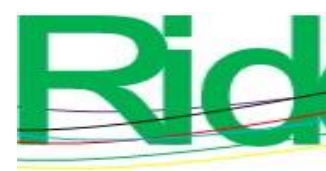

e

Revista Iberoamericana para la Investigación y el Desarrollo Educativo ISSN 2007 - 7467

En la tabla 3 se presenta la interpretación de la correlación de las variables de estudio, las cuales advierten un comportamiento diferente en cuanto a la presentación descriptiva, como se habían mostrado en la figura 2 y 3.

Tabla 3. Correlación de Pearson de variables de movilidad

\begin{tabular}{|l|c|c|}
\hline \multicolumn{1}{|c|}{ Variable } & $\begin{array}{c}\text { Por lugar de } \\
\text { movilidad }\end{array}$ & $\begin{array}{c}\text { Por medio de } \\
\text { movilidad }\end{array}$ \\
\hline Género & 0.8865 & 0.8865 \\
\hline Hogar & 0.9200 & 0.9200 \\
\hline Trabajo & 0.8967 & 0.8967 \\
\hline Estudio & 0.9971 & 0.9971 \\
\hline Compras & 0.7898 & 0.7898 \\
\hline Convivencia & 0.9528 & 0.9528 \\
\hline Llevar alguien & 0.7282 & 0.7282 \\
\hline Trámites & 0.9808 & 0.9808 \\
\hline Salud & 0.8944 & 0.8944 \\
\hline Religión & 0.8115 & 0.8115 \\
\hline Otro & 0.9990 & 0.9990 \\
\hline No sabe & 0.9687 & 0.9687 \\
\hline Total lugar & 1.0000 & 1.0000 \\
\hline Auto & & 0.9493 \\
\hline Otro medio & & 0.9972 \\
\hline & & 1.0000 \\
\hline
\end{tabular}

Fuente: Elaboración propia con base en Inegi (2017)

Se realizó un análisis de coeficiente de Pearson para medir el grado de correlación entre las variables en relación con la movilidad. Los resultados reflejaron lo siguiente: el género (0.8865) en relación con el propósito de ir al hogar (0.9200), ir al trabajo (0.8967), al lugar de estudio (0.9971), para ir de compras (0.7898), al lugar de convivencia (0.9528), para llevar a alguien a un destino (0.7282), para realizar trámites (0.9808), para acudir a un recinto de salud (0.8944) y para dirigirse a un lugar religioso (0.8115). 


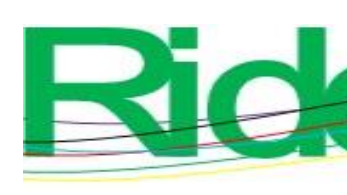

Revista Iberoamericana para la
Investigación y el Desarrollo Educativo
ISSN $2007-7467$

Se concluye que la correlación más alta es en el desplazamiento necesario para ir al lugar de estudio; enseguida, para acudir a lugares de convivencia, y el tercer motivo, dirigirse al hogar. Visualizando el comportamiento estadístico descriptivamente, es el trabajo el que ocupa el primer lugar, sigue el estudio y en tercer posicionamiento ir de compras. Por lo tanto, se debe administrar de una manera eficaz el uso del transporte público como medio directo en la movilidad del individuo para mejorar las condiciones.

La actuación femenina es muy activa; en el propósito de viaje tiene presencia primero en ir al trabajo, lugar de estudio e ir de compras. En cuanto al medio utilizado para moverse, sobrepasa, por mucho, el uso de otro medio diferente al auto particular. En el resultado sí hay coincidencia en los dos análisis, el descriptivo y coeficiente de Pearson, al utilizar otro medio de transporte diferente al auto particular (0.9972). Así pues, visualizando la participación tan representativa de la mujer, se propone considerar la formación humana, incidir de manera directa en la educación como agente de transformación hacia un respeto directo al prójimo, así como la administración eficiente hacia un transporte público eficaz.

Los resultados de esta investigación coinciden con los de otros estudios similares en donde el patrón rector es el trabajo y la educación y el desplazamiento por compras varias se posicionan enseguida de este.

A partir de los resultados se propone un diseño de administración considerando dos elementos o agentes activos, el administrador público y el usuario del servicio. Se parte de la corresponsabilidad en el proceso visto desde un enfoque sistémico (véase tabla 4 y figura 4 ).

\section{Propuesta}

Para este caso se sugiere, una vez teniendo la estadística específica de la movilidad cotidiana poblacional y a través de la planeación estratégica, seleccionar y proponer de manera eficiente y eficaz el uso del transporte, específicamente del transporte público. Se sugiere que con la utilización de un sistema ERP (por sus siglas en inglés, Enterprise Resource Planning) y el uso de tarjetas digitales o alguna app hace más eficaz el proceso sistémico, con el involucramiento corresponsable del administrador del servicio y del usuario.

A continuación, en la tabla 4 y figura 4 , se describe de manera sucinta la forma del proceso que se propone, tomando como referencia el diagnóstico y los resultados obtenidos del análisis de datos y considerando los diferentes participantes y roles de los mismos. 


\section{Revista Iberoamericana para la Investigación y el Desarrollo Educativo ISSN 2007 - 7467}

Tabla 4. Propuesta de administración corresponsable

\begin{tabular}{|l|l|c|}
\hline \multicolumn{1}{|c|}{ Actores } & \multicolumn{1}{|c|}{ Definición } & \multicolumn{1}{|c|}{ Elementos-acciones } \\
\hline Administración & Proceso & $\bullet$ Diagnóstico \\
de la autoridad & específico y & $\bullet$ Cadena de valor- \\
& oficial realizado & privilegiando al sujeto- \\
& por personal & usuario \\
& competente que & $\bullet$ ERP. Con su respectiva \\
& represente a la & reingeniería de procesos \\
& autoridad pública & \\
& oficial. & \\
\hline Administración & Proceso de & $\bullet$ Autogestión administrativa \\
del Usuario & autogestión de la & $\bullet$ Uso de ERP, como usuario- \\
& persona para & sugerido \\
& definir por & - Beneficios: uso de tarjetas \\
& prioridades su & digitales o apps \\
& movilidad. & (smartphone) \\
\hline
\end{tabular}

Fuente: Elaboración propia 


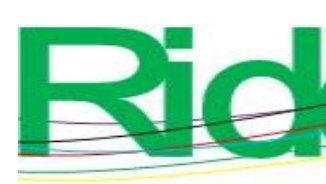

\section{Revista Iberoamericana para la Investigación y el Desarrollo Educativo ISSN 2007 - 7467}

Figura 4. Proceso de administración-corresponsable

Proceso administrador

$$
\text { I Diagnóstico }\left\{\begin{array}{l}
\text { - Unidades disponibles- Tipo de vehiculos } \\
\text { II Duario- población- propósito del viaje- } \\
\text { número de viajes- horario de uso }
\end{array}\right.
$$

Fuente: Elaboración propia

\section{Discusión}

La movilidad, una necesidad apremiante del individuo ante el crecimiento de las ciudades que han sobrepasado sus límites, requiere de un proceso administrativo muy específico. Para ello, es necesario conocer los principales motivos por los que las personas necesitan o desean desplazarse de manera cotidiana.

En Suiza, por ejemplo, los viajes que realizan las personas están marcados por especificidades estructurales vinculadas al mercado laboral, especificidades institucionales asociadas a un estado federalista y a un contexto específico. Destaca la alta movilidad espacial relacionada con el trabajo de la población de entre 25 a 54 años (11\%). La tasa varía y asciende a $13 \%$ con ocupación parcial y $15 \%$ con ocupación a tiempo completo. Esta última proporción se debe principalmente a que la población que trabaja a tiempo parcial en su mayoría son mujeres (84\%), ya que deben combinar el trabajo y la familia (Viry, Kaufmann y Widmer, 2009). Esta última condición se puede vincular con los datos arrojados en esta investigación: la mujer participa activamente en la movilidad familiar. 


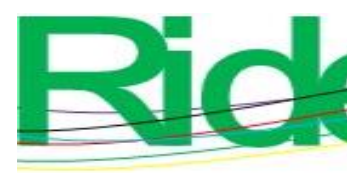

Revista Iberoamericana para la Investigación y el Desarrollo Educativo ISSN $2007-7467$

De manera general, los individuos tienen la oportunidad de elegir entre un amplio abanico de modos de desplazamiento y un sistema de transporte extenso, sin embargo, variables como la edad, el género, el nivel de renta o las capacidades físicas e intelectuales influyen al momento de trasladarse de un punto a otro (Montouto y Yustos, 2010). La movilidad y el transporte colectivo deficiente inciden en la exclusión social (García, 2014). Cabe mencionar que en un estudio realizado en España los traslados al trabajo en vehículos privados representaron $40 \%$ de la movilidad total en un día laborable (Cremades y Gómez, 2019). Esta situación también se refleja en este trabajo.

Aquí lo más preocupante ha sido la planeación del transporte como infraestructura desvinculada al crecimiento de las ciudades y la falta de mejoría de la oferta en las periferias de baja densidad. Es de subrayar el hecho de que los usuarios constantemente se quejan de los tiempos de espera para tomar el transporte $(19.63 \%)$ y, algo íntimamente relacionado con lo anterior, de la poca frecuencia del transporte público (17.47 \%) (Aón et al., 2017). Al respecto, García (2014) menciona que la disminución de este factor, el tiempo de espera, puede lograr que los tiempos de traslado en transporte público se aproximen a los del auto particular e incentiva el uso del transporte masivo.

\section{Conclusiones}

La movilidad como una necesidad natural del ser humano se manifiesta en los desplazamientos rutinarios que realizamos de manera cotidiana para satisfacer nuestras necesidades: motivos de trabajo, escolar, salud, recreación, entre otros.

Los datos resultantes coinciden con la información plasmada en estudios similares, en donde el eje rector es el trabajo como necesidad primaria, y continua el desplazamiento por motivos de educación y posteriormente la movilidad por compras varias.

El objetivo propuesto se logró en su totalidad al identificar los motivos principales de la movilidad cotidiana de los individuos de una zona específica, las causas de los desplazamientos con base en los propósitos de que generan sus viajes y la precisión del tipo de transporte utilizado. Un aspecto muy importante fue señalar y reconocer el involucramiento de la mujer en estas acciones y resaltar el papel tan notable que ejerce en la parte axiológica, y así poder identificar la corresponsabilidad desde el proceso administrativo (autogestivo), el comportamiento y respeto al prójimo, el cuidado de los servicios y desde luego el impacto que ello tiene en nuestro entorno. 


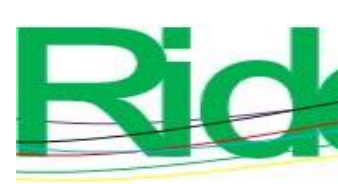

Revista Iberoamericana para la
Investigación y el Desarrollo Educativo
ISSN $2007-7467$

Otro aspecto troncal fue el poder considerar la movilidad (motivos) cotidiana para diseñar una propuesta de administración del transporte público que impacte de manera positiva en la dinámica de los usuarios para optimizar los tiempos y movimientos de la comunidad. Esto dio pauta para que, con base en el análisis estadístico, se pudiera enfatizar en el rechazo de la hipótesis nula y se aceptara la alterna, ya se corroboró que sí existe una correlación de los motivos de desplazamiento cotidiano con la movilidad.

Se considera pertinente tener muy en claro los motivos que provocan los desplazamientos cotidianos, así como la frecuencia de ellos; todo esto para tener un diagnóstico objetivo y puntual de la movilidad cotidiana de la población, para administrar el transporte y desde luego enfatizar en el público.

Para este caso se sugiere que, una vez teniendo la estadística específica de la movilidad cotidiana poblacional y a través de la planeación estratégica, seleccionar y proponer de manera eficiente y eficaz el uso del transporte y específicamente del transporte público. En este sentido, la utilización de un sistema ERP, combinado con el uso de tarjetas digitales o apps, puede ser clave para un proceso sistémico eficaz, con el involucramiento corresponsable del administrador del servicio y del usuario.

\section{Futuras líneas de investigación}

Una de las principales limitantes fue que el análisis únicamente consideró el viaje de un primer tramo; sería conveniente realizarlo considerando distancias mayores o viajes completos.

También es pertinente trabajar en el aspecto administrativo del transporte ante la pandemia, ya que la parte de conciencia de los usuarios debe de accionarse de manera volitiva.

Es muy recomendable también vincular la movilidad con los costos, precios por transporte utilizado, y en relación con las distancias que debe recorrer en sus desplazamientos y con el tiempo que le conlleva esa movilidad cotidiana.

Una recomendación muy importante es trabajar de manera específica el rol tan importante que tiene la mujer en la determinación de los motivos para realizar los desplazamientos, en la administración de los tiempos y recursos dentro del hogar y su rol como trabajadora fuera del hogar. También resaltar el vínculo directo en la comunicación y transmisión de valores hacia los hijos. 


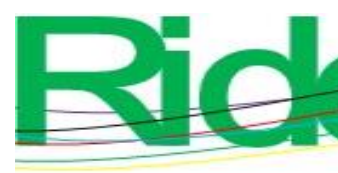

Revista Iberoamericana para la Investigación y el Desarrollo Educativo ISSN $2007-7467$

Un aspecto apremiante que debe seguirse trabajando, también vinculado al género, es el hacinamiento colectivo por la pandemia. Al respecto, la Organización Internacional del Trabajo (OIT) menciona que en tiempos regulares el trabajo de la mujer en el hogar era tres veces mayor al del hombre. En el contexto de pandemia por la COVID-19, la condición de la mujer se ha agravado al grado tal que ha tenido que asumir múltiples responsabilidades simultáneas (Comisión Económica para América Latina y el Caribe [Cepal]-Organización de las Naciones Unidas para la Educación, la Ciencia y la Cultura [Unesco], 2020).

\section{Agradecimientos}

Al Instituto Politécnico Nacional por su apoyo para la realización del proyecto SIP20201737, “Administración de recursos naturales en hogares en estado de pobreza”, del cual deriva este trabajo.

\section{Referencias}

Aón, L., López, M. J., Freaza, N., Cola, C. y Giglio, M. L. (2017). Observatorio de Movilidad Urbana Gran La Plata. Tomo 2. Argentina: Instituto de Investigación y Políticas del Ambiente Construido.

Aparicio, E. C. (2018). Movilidad cotidiana e infraestructura en la configuración del espacio rural no periurbano. Región y Sociedad, 30(71). Recuperado de https://doi.org/https://doi.org/10.22198/rys.2018.71.a399.

Boniolo, P. y Estévez, B. (2017). El efecto del territorio en la movilidad social de hogares de la Región Metropolitana de Buenos Aires. Cuadernos Geográficos, 56(1), 101-123. Recuperado de https://dialnet.unirioja.es/servlet/articulo?codigo=5960915.

Comisión Económica para América Latina y el Caribe [Cepal]-Organización de las Naciones Unidas para la Educación, la Ciencia y la Cultura [Unesco]. (2020). La educación en tiempos de la pandemia de COVID-19. Santiago, Chile: Comisión Económica para América Latina y el Caribe- Organización de las Naciones Unidas para la Educación, la Ciencia y la Cultura.

Centro de Estudios de las Finanzas Públicas [CEFP]. (2019). Aspectos relevantes del Plan Nacional de Desarrollo 2019-2024. Ciudad de México, México: Centro de Estudios de las Finanzas Públicas. Recuperado de https://www.cefp.gob.mx/publicaciones/documento/2019/cefp0112019.pdf 


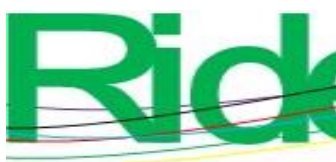

Revista Iberoamericana para la
Investigación y el Desarrollo Educativo
ISSN $2007-7467$

Cremades, L. y Gómez, M. (2019). Impacto en la salud y en el medioambiente de las medidas para incentivar la reducción del uso del vehículo privado por los trabajadores. ORP Journal, 2019(1), 103-111.

CTS Embarq México. (2015). Proyecto de transformación del transporte público concesionado. Ciudad de México, México: CTS Embarq México. Recuperado de https://theicct.org/sites/default/files/PresentacionSEDEMASITP_CTS EMBARQ.pdf.

Galindo, M. C., Pérez, E. y Suárez, M. (2020). Movilidad intrarregional en la región Centro de México, 2000-2015. Investigaciones Geográficas, 102. Recuperado de https://doi.org/10.14350/rig.60093.

Grupo IGN. (2020). Transporte público. Recuperado de https://ignsl.es/experiencia/transporte-publico/.

Gutiérrez, A. (2012). ¿Qué es la movilidad? Elementos para (re)construir las definiciones básicas del campo del transporte. Bitácora Urbano Territorial, 21(2), 61-74. Recuperado de https://dialnet.unirioja.es/servlet/articulo?codigo=5001899.

Gouëset, V., Demoraes, F., Le Roux, G., Figueroa, O. y Zioni, S. (2015). Recorrer la Metrópoli. Prácticas de movilidad cotidiana y desigualdades socio-territoriales en Bogotá, Santiago de Chile y São Paulo. En Dureau, F., Lulle, T., Souchaud, S. y Contreras, Y. (eds.), Movilidades y cambio urbano. Bogotá, Santiago y São Paulo (pp. 303-344). Bogotá, Colombia: Universidad Externado de Colombia.

H. Congreso de la Ciudad de México I Legislatura. (23 de abril de 2020). Decreto por el que se reforman y adicionan diversas disposiciones de la Ley de Movilidad del Distrito Federal. Gaceta Oficial de la Ciudad de México. Recuperado de http://legismex.mty.itesm.mx/estados/ley-df/DF-L-Movilidad-Ref2020_04.pdf.

Hernández, R., Fernández, C. y Baptista, M. (2014). Metodología de la investigación. Ciudad de México, México: McGraw-Hill.

Instituto Nacional de Estadística y Geografía [Inegi]. (2017). Encuesta Origen Destino en Hogares de la Zona Metropolitana del Valle de México (EOD) 2017. México: Instituto Nacional de Estadística y Geografía. Recuperado de https://www.inegi.org.mx/programas/eod/2017/.

Kaufmann, V. and Widmer, E. (2006). Motility and family dynamics: Current issues and research agendas. Zeitschrift für Familienforschung, 18(1), 111-129. 


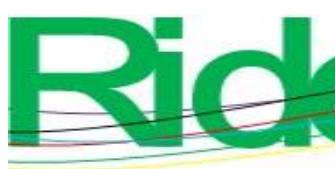

Revista Iberoamericana para la
Investigación y el Desarrollo Educativo
ISSN $2007-7467$

Kim, M., Kim, S. and Sohn, H. (2018). Relationship between Spatio-Temporal Travel Patterns Derived from Smart-Card Data and Local Environmental Characteristics of Seoul, Korea. Sustainability, 10(3).

Leicht, A., Heiss, J. and Byun, W. J. (eds.) (2018). Issues and Trends in Education for Sustainable Development. France, Paris: United Nations Educational, Scientific and Cultural Organization.

Long, Y. and Thill, J. C. (2015). Combining smart card data and household travel survey to analyze jobs-housing relationships in Beijing. Computers, Environment and Urban Systems, 53, 19 - 35.

Lyons, G. (2018). Getting smart about urban mobility - Aligning the paradigms of smart and sustainable. Transportation Research Part A: Policy and Practice, 115, 4-14. Retrieved from https://doi.org/10.1016/j.tra.2016.12.001.

Montouto, O. y Yustos, J. L. (2010). Guía educativa. Por una movilidad escolar, sostenible y segura. España: Diputación de Albacete. Recuperado de https://dialnet.unirioja.es/servlet/libro?codigo $=740339$.

Paredes, A. M. y Palmer, M. C. A. (2018). Nivel de satisfacción de los usuarios de transporte público colectivo en la Ciudad de México aplicando el enfoque agregado. Administración y Organizaciones, 21(40), 119-135. Recuperado de https://rayo.xoc.uam.mx/index.php/Rayo/article/view/21

Pérez, R. y Capron, G. (2018). Movilidad cotidiana, dinámicas familiares y roles de género: análisis del uso del automóvil en una metrópoli latinoamericana. Quid 16: Revista del Área de Estudios Urbanos, (10), 102-128. Recuperado de https://dialnet.unirioja.es/servlet/articulo?codigo $=6702384$.

Real Academia Española [RAE]. (2020). Movilidad. En Diccionario de la lengua española (23. ${ }^{\mathrm{a}}$ ed.). Recuperado de https://dle.rae.es/movilidad?m=form.

Riquelme, H. (2016). Movilidad cotidiana: entre la producción y reproducción social. Una exploración a las prácticas de desplazamiento de dos mujeres en Temuco. Pilquen, 19(4), 14-31. Recuperado de https://dialnet.unirioja.es/servlet/articulo?codigo $=5763453$.

Tang, J., Wang, X., Zong, F. and Hu, Z. (2020). Uncovering Spatio-temporal Travel Patterns Using a Tensor-based Model from Metro Smart Card Data in Shenzhen, China. DPI, Open Access Journal, 12(4), 1-16. 


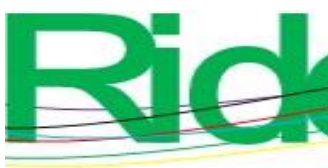

Revista Iberoamericana para la Investigación y el Desarrollo Educativo ISSN 2007 - 7467

Viry, G., Kaufmann, V. and Widmer, E. D. (2009). Switzerland - Mobility: a life stage issue? In Schneider, N. F. and Meil, G. (eds.), Mobile Living across Europe I: Relevance and Diversity of Job-Related Spatial Mobility in Six European Countries (pp. 189228). Barbara Budrich Verlag.

\begin{tabular}{|c|c|}
\hline Rol de Contribución & Autor(es) \\
\hline Conceptualización & $\begin{array}{l}\text { Ma. de los Ángeles Martínez Ortega <igual>, Laura Vázquez } \\
\text { Nájera <igual>, Martha Jiménez García<igual> }\end{array}$ \\
\hline Metodología & Ma. de los Ángeles Martínez Ortega \\
\hline Software & $\begin{array}{l}\text { Martha Jiménez García<igual>Ma. de los Ángeles Martínez } \\
\text { Ortega <igual>, }\end{array}$ \\
\hline Validación & $\begin{array}{l}\text { Ma. de los Ángeles Martínez Ortega <igual>, Laura Vázquez } \\
\text { Nájera <igual>, Martha Jiménez García<igual> }\end{array}$ \\
\hline Análisis Formal & Ma. de los Ángeles Martínez Ortega \\
\hline Investigación & $\begin{array}{l}\text { Ma. de los Ángeles Martínez Ortega <igual>, Laura Vázquez } \\
\text { Nájera <igual>, Martha Jiménez García<igual> }\end{array}$ \\
\hline Recursos & Ma. de los Ángeles Martínez Ortega \\
\hline Curación de datos & $\begin{array}{l}\text { Ma. de los Ángeles Martínez Ortega <igual>, Laura Vázquez } \\
\text { Nájera <igual>, Martha Jiménez García<igual> }\end{array}$ \\
\hline $\begin{array}{l}\text { Escritura - Preparación del } \\
\text { borrador original }\end{array}$ & $\begin{array}{l}\text { Ma. de los Ángeles Martínez Ortega <igual>, Laura Vázquez } \\
\text { Nájera <igual>, Martha Jiménez García<igual> }\end{array}$ \\
\hline $\begin{array}{l}\text { Escritura - Revisión y } \\
\text { edición }\end{array}$ & Ma. de los Ángeles Martínez Ortega \\
\hline Visualización & Ma. de los Ángeles Martínez Ortega \\
\hline Supervisión & Ma. de los Ángeles Martínez Ortega \\
\hline Administración de Proyectos & Ma. de los Ángeles Martínez Ortega \\
\hline Adquisición de fondos & Ma. de los Ángeles Martínez Ortega \\
\hline
\end{tabular}

